\title{
A NEW NETWORK FOR HIGHER-TEMPERATURE GAS-PHASE CHEMISTRY. I. A PRELIMINARY STUDY OF ACCRETION DISKS IN ACTIVE GALACTIC NUCLEI
}

\author{
Nanase Harada ${ }^{1}$, ERIC Herbst ${ }^{2}$, and VAlentine WaKelam ${ }^{3,4}$ \\ ${ }^{1}$ Department of Physics, The Ohio State University, Columbus, OH 43210, USA \\ 2 Departments of Physics, Astronomy, and Chemistry, The Ohio State University, Columbus, OH 43210, USA \\ 3 Université de Bordeaux, OASU, BP 89, F-33271 Floirac Cedex, France \\ ${ }^{4}$ CNRS, UMR 5804, LAB BP 89, F-33271 Floirac Cedex, France \\ Received 2010 April 19; accepted 2010 August 5; published 2010 September 10
}

\begin{abstract}
We present a new interstellar chemical gas-phase reaction network for time-dependent kinetics that can be used for modeling high-temperature sources up to $\approx 800 \mathrm{~K}$. This network contains an extended set of reactions based on the Ohio State University (OSU) gas-phase chemical network. The additional reactions include processes with significant activation energies, reverse reactions, proton exchange reactions, charge exchange reactions, and collisional dissociation. Rate coefficients already in the OSU network are modified for $\mathrm{H}_{2}$ formation on grains, ion-neutral dipole reactions, and some radiative association reactions. The abundance of $\mathrm{H}_{2} \mathrm{O}$ is enhanced at high temperature by hydrogenation of atomic $\mathrm{O}$. Much of the elemental oxygen is in the form of water at $T \geqslant 300 \mathrm{~K}$, leading to effective carbon-rich conditions, which can efficiently produce carbon-chain species such as $\mathrm{C}_{2} \mathrm{H}_{2}$. At higher temperatures, $\mathrm{HCN}$ and $\mathrm{NH}_{3}$ are also produced much more efficiently. We have applied the extended network to a simplified model of the accretion disk of an active galactic nucleus.
\end{abstract}

Key words: astrochemistry - galaxies: nuclei - molecular data - molecular processes

\section{INTRODUCTION}

The gas-phase chemistry of interstellar molecules in cold cores of dense interstellar clouds has been modeled for almost 40 years (Herbst \& Klemperer 1973; Herbst \& Millar 2008). The models utilize time-dependent kinetics rather than thermodynamics because the chemistry is driven by non-LTE processes such as cosmic-ray ionization and most reactions are too slow to be of importance at the low temperatures, typically $10-20 \mathrm{~K}$. In fact, the reactions contained in the model networks for low-temperature studies must be exothermic or thermoneutral and must not contain activation energy barriers. These reactions are mainly ion-neutral, ion-electron, and radical-neutral processes (see, e.g., http://www.udfa.net/ and http://www.physics.ohio-state.edu/ eric/). But the interstellar medium is certainly not all cold. Elevated temperatures in the interstellar medium can be obtained via a variety of processes such as gravitational contraction, irradiation by UV photons, $\mathrm{X}$-rays, or high fluxes of cosmic rays, turbulence, and the passage of shock waves. In order to model the chemistry of portions of clouds at higher temperatures, such as hot cores in the range $100-300 \mathrm{~K}$, there is a need to include both weakly endothermic reactions and those with moderate activation energy barriers, often known as high-temperature reactions. Some reactions of these types have been included in modern studies of hot cores and corinos (Garrod et al. 2008) and these and others are listed in the RATE06 catalog (see http://www.udfa.net/). As the temperature gets still higher, more reactions of these types are needed to model the chemistry successfully.

Inner regions of protoplanetary disks can reach temperatures in excess of $1000 \mathrm{~K}$, and the chemistry of these regions has become a topic of extreme interest, with models by many authors (e.g., Markwick et al. 2002; Gorti \& Hollenbach 2008; Agúndez et al. 2008; Nomura et al. 2009; Woods \& Willacy 2009; Glassgold et al. 2009; Woitke et al. 2009). Agúndez et al. (2008) have discussed some of the high-temperature reactions in their protoplanetary disk model. Other authors have used the RATE06 catalog with some additions. High temperatures can also be reached in accretion disks of active galactic nuclei (AGNs), as discussed in Section 4. Many high-temperature reactions have been included in shock models, where temporary temperatures after the shock front passes can reach $4000 \mathrm{~K}$. Given the relatively short period of high temperature for most shocks, reactions involving $\mathrm{H}_{2}$ are of extreme importance even if they involve barriers. For example, gas-phase water can be produced by the hydrogenation of oxygen atoms (Elitzur \& de Jong 1978; Neufeld \& Dalgarno 1989; Bergin et al. 1998). Elevated temperatures in the interstellar medium can also be found in photon-dominated regions (PDRs) and X-ray-dominated regions (XDRs). Indeed, the modeling of PDRs remains a major topic of study (for model comparison, see Röllig et al. 2007), while XDRs are also of high interest (Maloney et al. 1996; Meijerink \& Spaans 2005; Stäuber et al. 2005; Meijerink et al. 2007).

Although it is clear that the chemistry of high-temperature regions has been studied, there appears to be no general gas-phase network involving both simple and complex molecules that can be used for different types of sources. In this paper, we present what we believe to be the first general network of this type. To create it, we extended our gas-phase Ohio State University (OSU) network (see http://www.physics.ohio-state.edu/ eric/) by the inclusion of reactions with barriers, and the addition and modification of other reactions so that we can model the chemistry properly up to $800 \mathrm{~K}$. The network is purely a gas-phase one, with the exception of the formation of molecular hydrogen, and so is not appropriate for hot cores, where much of the chemistry derives from surface processes on warming grains (Garrod et al. 2008).

The rest of this paper is organized as follows. We discuss the higher-temperature reaction network in Section 2 and briefly look at the major effects of higher temperatures in Section 3. We then report an application of the new network in Section 4: specifically, we look at the chemistry as a function of radius from the central black hole in an accretion disk of an AGN. We 
will report the results for an AGN accretion disk model with more detailed consideration of varying physical conditions in a future paper (N. Harada et al. 2010, in preparation). Finally, a brief summary of our results is given in Section 5 .

\section{HIGHER-TEMPERATURE NETWORK}

The OSU gas-phase reaction network is designed for a cold interstellar cloud $(<100 \mathrm{~K})$. Unlike the OSU gas-grain code (Garrod et al. 2008), which contains both gas-phase and grainsurface reactions, and is designed for somewhat higher temperatures, it contains only one grain-surface reaction, the formation of molecular hydrogen. We based the high-temperature extension on the gas-phase network because most grain-surface reactions will be much less efficient when the dust temperature is high. The latest OSU network without anions, osu.09.2008, which was used as our base, contains 449 species and 4457 reactions. In order to model the chemistry of higher-temperature environments, we first added reactions with moderate activation energy or endothermicity taken from the RATE06 file (http://www.udfa.net/) and our gas-grain code, mainly estimated to be of importance at temperatures through $300 \mathrm{~K}$. We then added a further list of similar reactions, typically with higher activation energies or endothermicities, thought to be useful at higher temperatures, including the reverse reactions of selected exothermic processes in the network. With the addition of these new reactions, several species become dramatically more abundant at higher temperatures (e.g., $\mathrm{H}_{2} \mathrm{O}, \mathrm{NH}_{3}$, and their protonated species $\mathrm{H}_{3} \mathrm{O}^{+}, \mathrm{NH}_{4}^{+}$), so that we were forced to include additional reactions involving these species, including some proton exchange reactions. Because the balance between atomic and molecular hydrogen is critical to the chemistry, we revised the rate of molecular hydrogen formation for $100 \mathrm{~K}$ $<T<800 \mathrm{~K}$ (see Section 2.1 for the role of dust and gas temperatures). In addition, for ion-neutral reactions involving polar neutral reactants, we incorporated a better approximation for the rate coefficients, useful throughout the temperature range $10-800 \mathrm{~K}$ for those reactions not yet studied in the laboratory (Woon \& Herbst 2009). We included new charge exchange reactions and collisional dissociation processes, as well as modifying the rate coefficients of some radiative association reactions, adding some cosmic-ray-induced photodissociation processes lacking in earlier networks, and revising the code to incorporate high radiation fields and cosmic ray fluxes. The complete extended reaction network has an additional 947 reactions and four additional species, and will be available on the OSU Web site (http://www.physics.ohio-state.edu/ eric). In addition, these reactions will also be included eventually in the KIDA online database (http://kida.obs.u-bordeaux1.fr/). Below we discuss the addition and modification of classes of reactions to produce the higher-temperature network. It should be noted that it is implicitly assumed that the dust and gas temperatures are both high, so that dust-surface ice chemistry does not play a major role. Quantitatively, both gas and dust temperatures should exceed $100 \mathrm{~K}$.

\section{1. $\mathrm{H}_{2}$ Formation}

In the interstellar medium, molecular hydrogen is produced more efficiently on grains via the recombination of two atoms than in the gas phase. The formation rate can be written as

$$
\frac{d n\left(\mathrm{H}_{2}\right)}{d t}=\frac{1}{2} n_{\mathrm{H}} v_{\mathrm{H}} n_{\text {grain }} \sigma_{\text {grain }} S(T) \epsilon,
$$

where $n_{\mathrm{H}}$ is the number density and $v_{\mathrm{H}}$ is the thermal velocity of hydrogen atoms, $n_{\text {grain }}$ is the number density of grains, $\sigma_{\text {grain }}$ is the cross section of a grain particle, $S$ is the sticking coefficient for a hydrogen atom striking a grain as a function of temperature, and $\epsilon$ is the recombination efficiency. Without $\epsilon$, the expression is simply one-half of the volume rate at which $\mathrm{H}$ atoms strike and stick to grains. For the sticking coefficient and the efficiency, we utilize graphite grains, since the recombination efficiency has not been studied in detail for silicates at high temperatures. The sticking coefficient is a function of both the gas temperature $T$ and the dust temperature $T_{\mathrm{d}}$. The results of numerical calculations are shown in Burke \& Hollenbach (1983), but we use the simple formula of Hollenbach \& McKee (1979):

$$
S=\left[1+0.04\left(T+T_{\mathrm{d}}\right)^{0.5}+2 \times 10^{-3} T+8 \times 10^{-6} T^{2}\right]^{-1},
$$

which is a close fit to the numerical calculations for both silicates and graphite. If we assume the gas and dust temperatures to be equal, then the sticking coefficient is $\approx 0.8$ at $10 \mathrm{~K}, \approx 0.5$ at $100 \mathrm{~K}$, and $\approx 0.1$ at $800 \mathrm{~K}$. Although reality is doubtless much more complex (Cuppen \& Hornekær 2008), for the recombination efficiency of the reaction, we utilized the calculated results of Cazaux et al. (2005) for graphite surfaces, which are based on both the Langmuir Hinshelwood and Eley Rideal mechanisms. According to these authors, the efficiency decreases with increasing dust temperature, remaining near unity to a dust temperature of $30 \mathrm{~K}$, then decreasing as the dust temperature increases to $100 \mathrm{~K}$, afterward remaining roughly constant at 0.2 from 100 to $800 \mathrm{~K}$ with equal contributions from the two mechanisms. When the dust temperature is higher than $800 \mathrm{~K}$, the recombination efficiency decreases by orders of magnitude.

\subsection{Exothermic Ion-dipole Reactions}

Exothermic ion-neutral reactions involving non-polar neutral species typically have rate coefficients governed by the temperature-independent Langevin rate coefficient $k_{\mathrm{L}}$, which is given by the expression (in esu-cgs units):

$$
k_{\mathrm{L}}=2 \pi e \sqrt{\frac{\alpha_{\mathrm{pol}}}{\mu}},
$$

where $e$ is the electronic charge, $\alpha_{\text {pol }}$ is the scalar (dipole) polarizability, and $\mu$ is the reduced mass. There are, of course, exceptions to this simple approximation, most spectacularly the reaction between $\mathrm{He}^{+}$and $\mathrm{H}_{2}$ (Anicich 1993).

If the neutral is polar, however, the long-range force is no longer a central one, and the analysis is more complex. A variety of approximations exist for the rate coefficients $k$, and these have been handled somewhat differently in the RATE06 and OSU networks, both of which use the general form

$$
k=\alpha(T / 300 \mathrm{~K})^{\beta} \exp -(\gamma / T) .
$$

In the absence of a barrier $(\gamma=0)$, only one term in the power law can be used to express the temperature dependence. From a few measurements (Rebrion et al. 1988) and simplified theories (Su \& Chesnavich 1982; Herbst \& Leung 1986), the rate coefficients are assumed to vary inversely with the square root of temperature in the range $10-300 \mathrm{~K}(\beta=-1 / 2)$.

For the wide temperature range discussed here, a semiempirical approach can be used that derives from the trajectory scaling model of Su \& Chesnavich (1982) as refined by Troe and collaborators in terms of effective dipoles and regions of 
accuracy (Maergoiz et al. 2009). The approach, which has also been described by Woon \& Herbst (2009), is based on the unitless parameter $x$, which is given by the expression

$$
x=\frac{\mu_{\mathrm{D}}}{\sqrt{2 \alpha_{\mathrm{pol}} k_{\mathrm{B}} T}},
$$

where $\mu_{\mathrm{D}}$ is the dipole moment of the neutral reactant and $k_{\mathrm{B}}$ is the Boltzmann constant. Note that the actual dipole moment is used because it is normally not very different from the effective dipole discussed by Maergoiz et al. (2009). The recipe involves the expressions

$$
\begin{gathered}
k / k_{L}=0.4767 x+0.6200(x \geqslant 2), \\
k / k_{L}=(x+0.5090)^{2} / 10.526+0.9754(x<2) .
\end{gathered}
$$

As $x \rightarrow 0$, the rate coefficient $k$ reduces to the Langevin value. Also, as $x$ gets larger, the temperature dependence eventually reduces to the simple $T^{-1 / 2}$ dependence used previously. To utilize Equations (5)-(7), we have modified the input for the network in order to incorporate the parameters needed to compute the ion-polar rate coefficients as functions of temperature. The modified input can be found on our Web site (http://www.physics.ohio-state.edu/ eric/). A complete list of needed polarizabilities and dipole moments for interstellar neutral species has been calculated and reported by Woon \& Herbst (2009). An alternative approach for reactions studied in the laboratory at one or more temperatures would be to scale the theoretical results so as to fit the experimental ones. Further details concerning methods for calculating ion-dipole reaction rate coefficients are discussed in a review article from an international team under the auspices of the International Space Science Institute (Wakelam et al. 2010).

\subsection{Treatment of $U V$ and $X$-ray Photons}

In the original OSU gas-phase model, the Draine (1978) interstellar UV-photon field of $\chi=1.7$ is typically used, and the self-shielding and cross-shielding effects involving the photodissociation of $\mathrm{H}_{2}$ and $\mathrm{CO}$ are calculated with the approximate method of Lee et al. (1996), which is estimated to be valid up to $\chi=1000$. We modified the model in order to include a variable radiation field, and, for $\chi$ greater than 1000 , we utilize the Meudon PDR code (Le Petit et al. 2006) to obtain more accurate photodissociation rates for $\mathrm{CO}$ and $\mathrm{H}_{2}$. Such high radiative fluxes can be encountered in the inner regions of protoplanetary disks and accretion disks in AGNs.

These regions may also be subject to high fluxes of $\mathrm{X}$ rays. X-rays can ionize atoms directly, which can cause doubly ionized species for heavier atoms via the Auger mechanism. The "photo-electrons" produced by this primary X-ray ionization cause secondary ionization, which actually dominates for atomic hydrogen and atomic helium (Maloney et al. 1996). In addition, the photo-electrons can internally generate UV photons, which can then photodissociate molecules. In the current model, we approximate the effects of $\mathrm{X}$-ray fluxes by varying the cosmicray ionization rate, if needed.

The cross sections for primary ionization, however, are different for cosmic rays and X-rays, so that a refined treatment of X-rays is preferable and will eventually be included (Maloney et al. 1996; Meijerink \& Spaans 2005).

\subsection{Radiative Association}

Radiative association, a low-density process in which two reactants stick together by the emission of radiation, has only rarely been studied in the laboratory (Gerlich \& Kaefer 1989). Rate coefficients for these reactions can be estimated by comparison with three-body association rates studied in the laboratory or by statistical theories (Bates \& Herbst 1988). Both methods indicate that the rate coefficients increase dramatically as the temperature decreases $(\beta<0$ in Equation (4)) until, in rare instances, the association is saturated, and occurs on every collision (Herbst 1985). When rotation can be considered as a classical variable and vibration as a quantum mechanical one, the theoretical value for $\beta$ using the so-called modified thermal approach is given by the expression

$$
\beta \approx-\frac{1}{2}\left(r_{1}+r_{2}\right),
$$

where $r_{1}$ and $r_{2}$ are the numbers of rotational degrees of freedom of the reactants (Bates \& Herbst 1988). For example, for two nonlinear rotors as reactants, the theoretical value of $\beta$ is -3.0 . The range of temperature in which this formula is accurate depends on the system; the heavier the reactants, the lower the lower limit of temperature becomes, so that eventually the expression is accurate even at $10 \mathrm{~K}$ unless saturation has set it. But, the heavier the reactants, the lower the temperature at which vibration can be treated classically, which leads to an even stronger inverse dependence on temperature for the rate coefficient. For those reactions in the OSU network in which the temperature dependence is determined by Equation (8), we maintain this temperature dependence through $800 \mathrm{~K}$. For those reactions with a weaker temperature dependence, we assume that Equation (8) also holds above $300 \mathrm{~K}$. As an example, the reaction

$$
\mathrm{C}_{4} \mathrm{H}_{3}^{+}+\mathrm{C}_{2} \mathrm{H}_{2} \longrightarrow \mathrm{C}_{6} \mathrm{H}_{5}^{+}+\mathrm{h} v
$$

has a somewhat surprising rate coefficient of nearly $k=1.0 \times$ $10^{-9} \mathrm{~cm}^{3} \mathrm{~s}^{-1}$ at $300 \mathrm{~K}$ due perhaps to partial saturation, based on measurements with varying densities (Anicich 1993; McEwan et al. 1999). For higher temperatures, our approximation gives the rate coefficient $k=1.0 \times 10^{-9}\left(\frac{T}{300}\right)^{-2.5} \mathrm{~cm}^{3} \mathrm{~s}^{-1}$ since the number of rotational degrees of freedom for $\mathrm{C}_{4} \mathrm{H}_{3}^{+}$is 3 and that of $\mathrm{C}_{2} \mathrm{H}_{2}$, which is linear, is 2 . More accurate values that take vibration into account will require detailed calculations on individual systems.

\subsection{Reactions with Potential Barriers}

The rate coefficient of a reaction with a potential barrier, whether it is an activation energy barrier or a barrier simply due to reaction endothermicity, is proportional to $\exp (-\gamma / \mathrm{T})$, a factor that diminishes the rate coefficient, especially at low temperatures. Such reactions can become quite competitive at temperatures that approach $\gamma$ in value, assuming a standard gaskinetic value of $10^{-10}-10^{-11} \mathrm{~cm}^{3} \mathrm{~s}^{-1}$ for the pre-exponential factor. Reactions involving the most important neutral reactant, $\mathrm{H}_{2}$, can become competitive for significantly lower temperatures because there is four orders of magnitude more of this species than any other neutral molecule. This four order-of-magnitude enhancement cancels the diminution in rate by a value for $\gamma$ of $\approx 9 \times$ the temperature. Thus, a reaction involving $\mathrm{H}_{2}$ with $\gamma \approx 2000 \mathrm{~K}$ can become significant for $T \geqslant 200 \mathrm{~K}$.

As discussed previously, some of the reactions with barriers were taken from those in the RATE06 network, found at http://www.udfa.net. In addition, reactions were added from a shock network (G. Pineau des Forêts 2008, private communication) so that the model can cover even higher temperatures. The 
shock network includes hydrogenation reactions such as

$$
\begin{gathered}
\mathrm{C}_{2}+\mathrm{H}_{2} \longrightarrow \mathrm{C}_{2} \mathrm{H}+\mathrm{H}(\gamma=1420 \mathrm{~K}), \\
\mathrm{C}_{2} \mathrm{H}+\mathrm{H}_{2} \longrightarrow \mathrm{C}_{2} \mathrm{H}_{2}+\mathrm{H}(\gamma=1300 \mathrm{~K}), \\
\mathrm{C}_{2} \mathrm{H}_{2}+\mathrm{H}_{2} \longrightarrow \mathrm{C}_{2} \mathrm{H}_{3}+\mathrm{H}(\gamma=32,600 \mathrm{~K}),
\end{gathered}
$$

the first two of which onset at rather low temperatures so that they should possibly be included even in hot core models (G. E. Hassel et al. 2010, in preparation). The hydrogenation of acetylene is not important, on the other hand, until temperatures far in excess of those considered here. Nevertheless, hydrogenation reactions leading to more saturated hydrocarbons were included. Additional hydrogenation reactions involving $\mathrm{H}_{2}$ and their backward reactions, involving atomic $\mathrm{H}$, for carbon-chain species (e.g., the families $\mathrm{C}_{n}, \mathrm{C}_{n} \mathrm{H}, \mathrm{C}_{n} \mathrm{H}_{2} ; n>2$ ) were also included. Except for $n=3$, where the barriers were explicitly calculated by D. Woon (2009, private communication), we used the same rate coefficients as for $n=2$. To the best of our knowledge, these reactions have not been used elsewhere. J. Cernicharo and E. Roueff (2009, private communication) provided a long list of reactions relevant for combustion systems; among those included are endothermic reactions involving atomic hydrogen. Because of the hydrogenation of hydrocarbons, new species $\mathrm{C}_{2} \mathrm{H}_{6}$ and $\mathrm{C}_{10} \mathrm{H}_{2}$ and their protonated species $\mathrm{C}_{2} \mathrm{H}_{7}^{+}$and $\mathrm{C}_{10} \mathrm{H}_{3}^{+}$were added to the network.

Although not critical for the systems studied here, when the temperature reaches at least $1000 \mathrm{~K}$, collisions between molecules can start to dissociate $\mathrm{H}_{2}$ and other species. For example, an $\mathrm{H}_{2}$ molecule with sufficient kinetic energy can dissociate another molecular hydrogen:

$$
\mathrm{H}_{2}+\mathrm{H}_{2} \longrightarrow \mathrm{H}_{2}+\mathrm{H}+\mathrm{H} \text {. }
$$

The energy required to dissociate $\mathrm{H}_{2}$ by collisions with molecular hydrogen, atomic hydrogen, electrons, helium atoms, and protons, for example, is $\gamma=52,000 \mathrm{~K}$. Such reactions are included despite their high endothermicity. Another reaction with high endothermicity included in the network is

$$
\mathrm{H}+\mathrm{CO} \longrightarrow \mathrm{OH}+\mathrm{C},
$$

where $\gamma=77,000 \mathrm{~K}$.

\subsection{Thermodynamic Considerations}

According to thermodynamics, the ratio of the forward $k_{\mathrm{f}}$ and backward $k_{\mathrm{b}}$ rate coefficients for any reactive system is related by the expression

$$
k_{\mathrm{f}} / k_{\mathrm{b}}=\exp (-\Delta G / T)
$$

where $\Delta G$, in units of temperature, is the change in the Gibbs free energy of the reaction. The Gibbs free energy is itself given by the relation

$$
\Delta G=\Delta H-T \Delta S,
$$

where $\Delta H$ is the enthalpy change and $\Delta S$ is the entropy change between products and reactants. The enthalpy change is equal to the energy change in the ideal gas approximation when the number of products equals the number of reactants, and the energy change is roughly the same as the potential difference between products and reactants for a thermal system, although both thermodynamic energy and enthalpy have small temperature dependences. For those forward-backward systems where there is sufficient thermodynamic information, we used this information either to confirm independent estimates or measurements of the forward and backward reaction rates, or to include the rate coefficient of a backward reaction if judged to be important. If the independent values of the forward and backward rates strongly violated the correct thermodynamic ratio, we looked carefully at the sources of information to decide which was superior.

\subsection{Proton and Charge Exchange Reactions}

At room temperature and above, $\mathrm{H}_{2} \mathrm{O}, \mathrm{HCN}$, and $\mathrm{NH}_{3}$ are produced more abundantly than in low-temperature clouds. They have higher proton affinities than $\mathrm{CO}$ and $\mathrm{H}_{2}$, and so proton exchange reactions lead to high abundances of the protonated neutrals. At high temperatures, though, endothermic proton exchange reactions involving especially $\mathrm{H}_{3} \mathrm{O}^{+}$and $\mathrm{HCNH}^{+}$can be important, although few have been studied in the laboratory. To obtain their rate coefficients, we used the theoretical values for the exothermic direction discussed in Section 2.2 and then the thermodynamic ratio between forward and backward rate coefficients, discussed in the previous section. Unfortunately, entropy changes are not normally available, and we are forced to make the crude approximation that the change in free energy equals the change in enthalpy, which can be obtained from heats of formation or proton affinities.

As an example, consider the reaction system

$$
\mathrm{H}_{3} \mathrm{O}^{+}+\mathrm{HCN} \leftrightarrows \mathrm{HCNH}^{+}+\mathrm{H}_{2} \mathrm{O}
$$

which is exothermic in the forward direction with a decrease in enthalpy of $21.9 \mathrm{~kJ} \mathrm{~mol}^{-1}$ or $2600 \mathrm{~K}$ (Mallard et al. 2010). The backward (endothermic) reaction is slower than the forward one, but still occurs at high temperature. For example, at $800 \mathrm{~K}$, the ratio between the backward and forward rate coefficients arising from the Boltzmann factor is 0.04 . The abundant protonated species can also charge exchange with neutral metals to enhance the abundance of metallic ions, namely,

$$
\mathrm{NH}_{4}^{+}+\mathrm{Fe} \longrightarrow \mathrm{NH}_{3}+\mathrm{Fe}^{+}+\mathrm{H} \text {. }
$$

In this example, the initial product molecular neutral $\left(\mathrm{NH}_{4}\right)$ is unstable and dissociates.

\section{MAJOR EFFECTS OF HIGHER TEMPERATURES}

To get a feeling for the chemical effects that occur as the temperature is raised from $100 \mathrm{~K}$ to $800 \mathrm{~K}$ at constant density, we briefly present some results of model calculations. All results in this section are obtained using the pseudo-time-dependent model, in which chemical abundances evolve under fixed and homogeneous physical conditions. The fixed total hydrogen nuclear density here is $n_{\mathrm{H}}=2 \times 10^{4} \mathrm{~cm}^{-3}$, the visual extinction is $A_{V}=10$, and the standard interstellar radiation field is used. The cosmic-ray ionization rate $\zeta_{\mathrm{H}_{2}}$ is set at $1.3 \times 10^{-17} \mathrm{~s}^{-1}$. The initial abundances, shown in Table 1, are atomic except for $\mathrm{H}_{2}$ and reflect the so-called low metal oxygen-rich abundances used to obtain the correct ionization balance in dark clouds at $10 \mathrm{~K}$ (Graedel et al. 1982). The models with these parameters and physical conditions are termed "standard." Models for AGN accretion disks, discussed later, will utilize different parameters and physical conditions. 


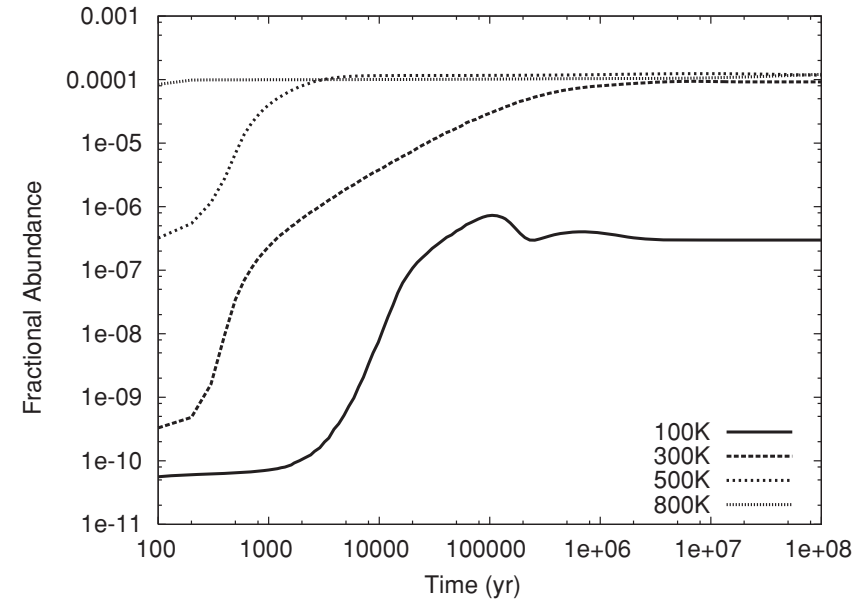

Figure 1. Fractional abundance of water as a function of time for a cloud with standard conditions at various temperatures from $100 \mathrm{~K}$ to $800 \mathrm{~K}$.

Table 1

Initial Fractional Abundances with Respect to Total Hydrogen

\begin{tabular}{lc}
\hline \hline Species & Low Metal Value \\
\hline $\mathrm{H}_{2}$ & 0.5 \\
$\mathrm{He}$ & 0.14 \\
$\mathrm{O}$ & $1.76(-4)$ \\
$\mathrm{N}$ & $2.14(-5)$ \\
$\mathrm{F}$ & $2.0(-8)$ \\
$\mathrm{Cl}$ & $3.0(-9)$ \\
$\mathrm{C}^{+}$ & $7.3(-5)$ \\
$\mathrm{Fe}^{+}$ & $3.0(-9)$ \\
$\mathrm{Mg}^{+}$ & $3.0(-9)$ \\
$\mathrm{Na}^{+}$ & $3.0(-9)$ \\
$\mathrm{P}^{+}$ & $3.0(-9)$ \\
$\mathrm{S}^{+}$ & $2.0(-8)$ \\
$\mathrm{Si}^{+}$ & $3.0(-9)$ \\
\hline
\end{tabular}

One major effect that does not occur is the destruction of $\mathrm{H}_{2}$. Rather, this species is still dominant at $800 \mathrm{~K}$, even if the efficiency of its formation on grains is not as high as in lowertemperature models. One of the effects of higher temperatures that does have a very large impact on the chemistry is the production of water via the hydrogenation reactions (Elitzur \& de Jong 1978; Neufeld \& Dalgarno 1989; Bergin et al. 1998)

$$
\begin{gathered}
\mathrm{O}+\mathrm{H}_{2} \longrightarrow \mathrm{OH}+\mathrm{H}(\gamma=3160 \mathrm{~K}) \\
\mathrm{OH}+\mathrm{H}_{2} \longrightarrow \mathrm{H}_{2} \mathrm{O}+\mathrm{H}(\gamma=1040 \mathrm{~K}) .
\end{gathered}
$$

By $300 \mathrm{~K}$, this sequence makes water the dominant form of oxygen beyond a certain time that decreases with increasing temperature, when it reaches a fractional abundance of $\approx 10^{-4}$ with respect to $n_{\mathrm{H}}$, as can be seen in Figure 1. As the temperature increases, the abundance of atomic oxygen becomes much smaller, while that of $\mathrm{CO}$, as shown in Figure 2, remains high although somewhat smaller than the elemental abundance of carbon. The remainder of the carbon goes mainly into $\mathrm{HCN}$ and acetylene. The lack of atomic oxygen has a significant impact on the carbon-chain chemistry. At temperatures below $100 \mathrm{~K}$, reactions with atomic oxygen are the dominant destruction of long carbon chains into shorter ones (e.g., $\mathrm{C}_{4} \mathrm{H}+\mathrm{O} \longrightarrow \mathrm{CO}+$ $\mathrm{C}_{3} \mathrm{H}$ ), which is one reason why the steady-state abundances of long carbon chains are much lower with oxygen-rich elemental abundances than with carbon-rich ones.

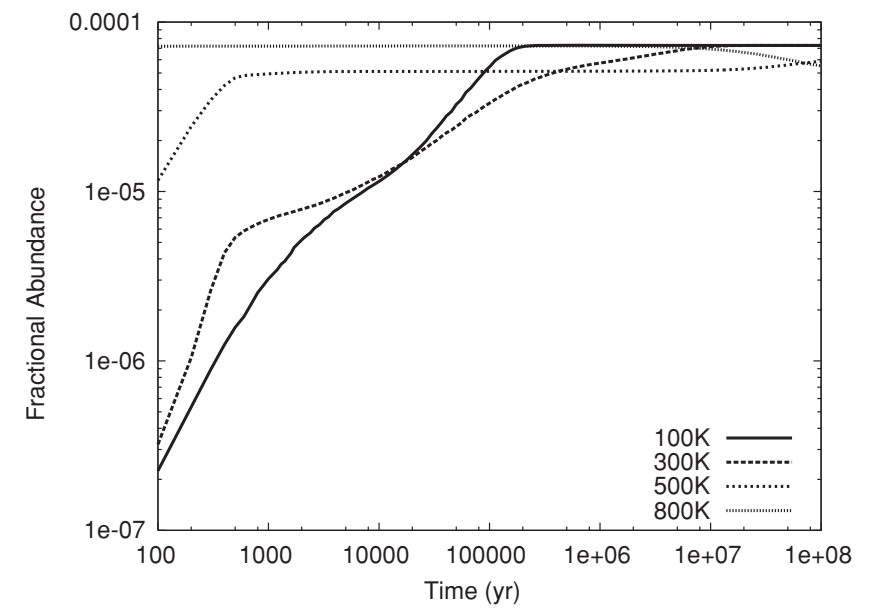

Figure 2. Fractional abundance of $\mathrm{CO}$ as a function of time for a cloud with standard conditions at various temperatures from $100 \mathrm{~K}$ to $800 \mathrm{~K}$.

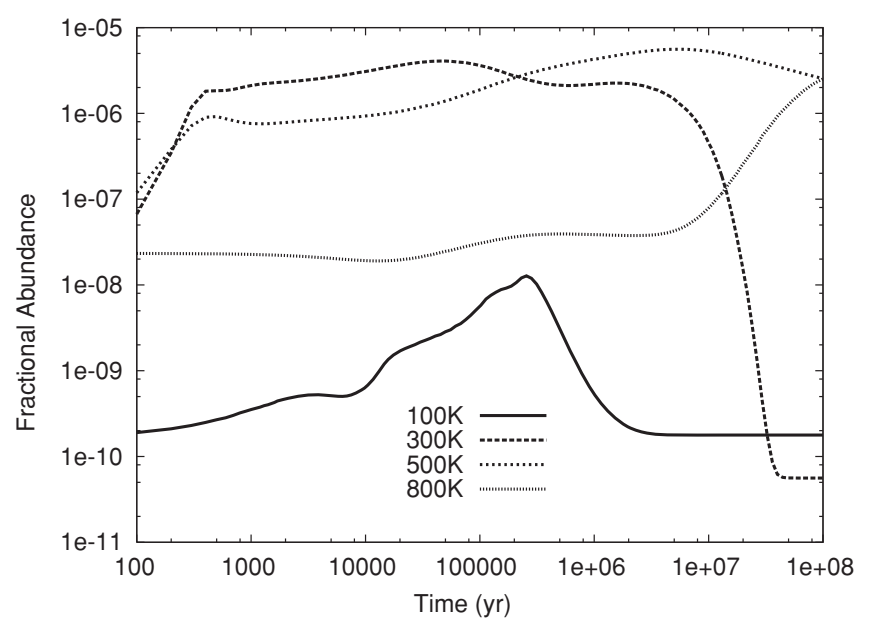

Figure 3. Fractional abundance of $\mathrm{C}_{2} \mathrm{H}_{2}$ as a function of time for a cloud with standard conditions at various temperatures from $100 \mathrm{~K}$ to $800 \mathrm{~K}$.

At higher temperatures, because of the lack of atomic oxygen, long carbon chains are not destroyed as efficiently, which makes the system effectively "carbon-rich." These carbon chains can also achieve a somewhat higher degree of saturation when the temperature is high enough to allow fast hydrogenation reactions with barriers. For example, a large amount of $\mathrm{C}_{2} \mathrm{H}_{2}$ is produced by reactions (10) and (11); the result can be seen in Figure 3. The time dependence of the abundance at $100 \mathrm{~K}$ is similar to the one in a typical O-rich system at $10 \mathrm{~K} ; \mathrm{C}_{2} \mathrm{H}_{2}$ reaches a peak abundance at the so-called early time and then declines. At $300 \mathrm{~K}$, the abundance is actually enhanced at early time but still decreases considerably toward steady state. By $500 \mathrm{~K}$, however, there is only a slight decrease from the high abundance at early time $\left(\geqslant 10^{-6}\right)$ to that at steady state. At still higher temperatures, the early-time abundance drops but the steady-state abundance remains high, so that by $800 \mathrm{~K}$, it is near $10^{-6}$. The long time scales to reach steady state at least partially derive from a remnant formation mechanism starting from the reaction between $\mathrm{CO}$ and $\mathrm{He}^{+}$. Further hydrogenation of acetylene does not occur even at $800 \mathrm{~K}$ because the activation energy barrier is too large. The story is similar for larger carbon-chain species, such as $\mathrm{C}_{9} \mathrm{H}_{2}$, which is produced from $\mathrm{C}_{9}$ and $\mathrm{C}_{9} \mathrm{H}$ via hydrogenation reactions with $\mathrm{H}_{2}$ at high temperature and is not destroyed readily by reaction with $\mathrm{H}_{2}$. The abundance of methane is also enhanced, although the change is not as extreme as the case of acetylene. 


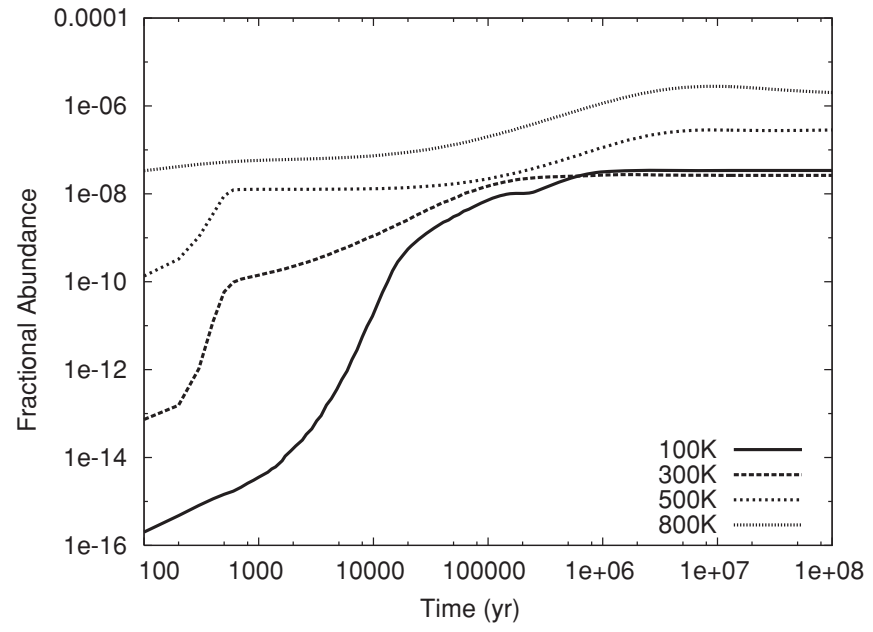

Figure 4. Fractional abundance of $\mathrm{NH}_{3}$ as a function of time for a cloud with standard conditions at various temperatures from $100 \mathrm{~K}$ to $800 \mathrm{~K}$.

Ammonia is enhanced over its abundance at low temperatures, as shown in Figure 4, reaching a fractional abundance of $\approx 3 \times 10^{-6}$ at $800 \mathrm{~K}$, which is only a factor of a few below the abundance of $\mathrm{N}_{2}$. Hydrogenation with $\mathrm{H}_{2}$ is important in forming $\mathrm{HCN}$ from $\mathrm{CN}$ with a relatively low reaction barrier of $\gamma=820 \mathrm{~K}$, which enhances the HCN abundance at high temperatures:

$$
\mathrm{CN}+\mathrm{H}_{2} \longrightarrow \mathrm{HCN}+\mathrm{H} \text {. }
$$

As discussed in Section 2.7, the protonated ions of the dominant high-temperature species such as $\mathrm{H}_{2} \mathrm{O}, \mathrm{HCN}$, and $\mathrm{NH}_{3}$ tend to become the most abundant ions at high temperatures, replacing $\mathrm{HCO}^{+}$and $\mathrm{H}_{3}^{+}$. While at $100 \mathrm{~K}, \mathrm{HCO}^{+}$takes up much of the positive charge at times after early time, by $300 \mathrm{~K}$, its relative abundance is sharply reduced. At this temperature, $\mathrm{H}_{3} \mathrm{O}^{+}$dominates at long times, with nearly half of the ionic abundance, and at $500-800 \mathrm{~K}, \mathrm{HCNH}^{+}$dominates at these times. Therefore, it is critical to include reactions with $\mathrm{H}_{3} \mathrm{O}^{+}$ and $\mathrm{HCNH}^{+}$in the network.

\section{MOLECULAR DISKS IN ACTIVE GALACTIC NUCLEI}

The term AGN refers to the center of a galaxy that is characterized by very high luminosity caused by the accretion of mass into a supermassive black hole. An AGN is also associated with an accretion disk consisting of a dusty torus, a few pc in extent, and a molecular disk that stretches out to hundreds of parsecs. Several molecular species have been observed in these disks on a $100 \mathrm{pc}$ scale, such as $\mathrm{CO}, \mathrm{HCN}, \mathrm{HCO}^{+}, \mathrm{HNC}$, and CN (see, for example, Schinnerer et al. 2000; Usero et al. 2004; Tacconi et al. 1994; Aalto 2008). These observational results have been compared with both PDR and XDR models. For example, Meijerink \& Spaans (2005) constructed models for both cases, Meijerink (2006) analyzed different CO transitions and the $\mathrm{HCO}^{+} / \mathrm{HCN}$ intensity ratio in different types of galaxies to see whether UV photons or X-rays have the dominant effect, and a larger set of observational results was compared with grid models by Meijerink et al. (2007). From such work, relative intensities of CO lines from a typical AGN, NGC 1068, can be explained by XDR scenarios while the $\mathrm{HCO}^{+} / \mathrm{HCN}(1-0)$ ratio fits better with a PDR model and can be fit by an XDR model only when the total column density absorbing the X-ray source is low. The observations of $\mathrm{CN}, \mathrm{HCN}$, and $\mathrm{HNC}$ by PérezBeaupuits et al. (2007) indicate that the $\mathrm{CN} / \mathrm{HCN}$ and $\mathrm{HNC} /$ $\mathrm{HCN}$ ratios favor PDR models more than XDR models, although the observed intensity may also come from star formation at a larger distance from the AGN than the circumnuclear disk of a few hundred pc. More recent observations of these molecules at higher $J$ lines also do not give a conclusive result as to whether the emission favors a PDR or an XDR (Pérez-Beaupuits et al. 2009).

On the scale of molecular observations, the gas temperature in the disks is under $100 \mathrm{~K}$, but it must be much higher when it is closer to the core of an AGN. To obtain a sense of the range of temperatures, we utilize the blackbody approximation, in which the temperature $T$ at $r$ pc from a central black hole of luminosity $L_{\mathrm{AGN}}$ is given by the expression

$$
T \sim 7.5 \times 10^{2} \mathrm{~K}\left(\frac{r}{\mathrm{pc}}\right)^{-1 / 2}\left(\frac{L_{\mathrm{AGN}}}{2 \times 10^{45} \mathrm{erg} \mathrm{s}^{-1}}\right)^{1 / 4},
$$

where the luminosity is normalized to that of NGC 1068, a prototypical AGN-containing galaxy with an intrinsic AGN luminosity, $L_{\mathrm{AGN}}$, of $2 \times 10^{45} \mathrm{erg} \mathrm{s}^{-1}$ (Mason et al. 2006). Thus, for an AGN with the luminosity of NGC 1068, the temperature at $1 \mathrm{pc}$ from the center is $750 \mathrm{~K}$ whereas that at $100 \mathrm{pc}$ from the center is $75 \mathrm{~K}$. Note that in normalizing the luminosity, we have not committed ourselves to either the PDR or XDR viewpoint.

The blackbody approach used here is rather approximate. The luminosity for NGC 1068 was actually obtained by fitting a clumpy model to observational data on the dusty torus in NGC 1068 (Mason et al. 2006; Nenkova et al. 2008), which shows the dust temperature to be $800 \mathrm{~K}$ in the inner sub-pc torus and $300 \mathrm{~K}$ for the few-pc-sized torus (Jaffe et al. 2004; Raban et al. 2009). This high contrast can be explained if it is assumed that higher temperatures come from the end of each clumpy surface that lies closer to the AGN while the lower temperatures must come from the other end. Our blackbody approximation as a function of radius would lie somewhere in between the value of $T_{\max }$ and $T_{\min }$ in Nenkova et al. (2008) for each clump.

\subsection{Physical Conditions in the Midplane}

In this paper, we simply use the blackbody approximation for the gas temperature with $L_{\mathrm{AGN}}=2 \times 10^{45} \mathrm{erg} \mathrm{s}^{-1}$. The mass density $\rho$ of the midplane of the accretion disk is given by the expression (Thompson et al. 2005)

$$
\rho=\frac{\Omega^{2}}{\sqrt{2} \pi G Q},
$$

where the rotation frequency $\Omega=\Omega_{K}$, the local Keplerian frequency, is defined by

$$
\Omega=\sqrt{\frac{G M_{\mathrm{BH}}}{r^{3}}+\frac{2 \sigma^{2}}{r^{2}}},
$$

in which $M_{\mathrm{BH}}$ is the mass of the black hole, $\sigma$ is the velocity dispersion, and $r$ is the distance from the AGN core. The Toomre stability parameter $Q$, a parameter for star formation regulation developed by Toomre (1964), is expressed as

$$
Q \equiv \frac{v_{s} \kappa}{\pi G \Sigma}
$$

for a gaseous disk, where $v_{s}$ is the sound speed, $\kappa$ is the epicyclic frequency, and $\Sigma$ is the surface density. When the value of $Q$ is small, the velocity dispersion is small and the disk is unstable to gravitational collapse, while a large value of $Q$ means that 
the disk is stable. The models of self-regulated star formation argue that $Q \sim 1$ in galactic disks since too high a value of $Q$ will cool the gas to reduce the dispersion of the gas, which leads to a lower value of $Q$, while too low a value of $Q$ will boost star formation to heat the gas leading to higher $Q$ (Silk 1997; Thompson et al. 2005). Observational studies (Martin \& Kennicutt 2001; Koda et al. 2005; Lucero \&Young 2007) show that $Q \sim 0.5-4$ for spiral galaxies. Vollmer et al. (2008) argue that depending on the evolutional stage of the galaxy, the Toomre $Q$ parameter can be higher than the order of unity, e.g., $Q \sim 190$ at the Galactic Center, but their AGN samples show smaller deviations from unity, with $Q=4-6$. Here, we make an assumption that $Q=1$ in Equation (23) to obtain $\rho$ and then obtain the midplane number density $n_{\mathrm{H}}$ from $n_{\mathrm{H}}=\frac{\rho}{m_{\mathrm{H}}}$, where $m_{\mathrm{H}}$ is the proton mass. In this paper, we use $M_{\mathrm{BH}}=1 \times 10^{7} M_{\odot}$, which is a reasonable value for $M_{\mathrm{BH}}$ in NGC 1068 (Greenhill \& Gwinn 1997; Tremaine et al. 2002), and $\sigma=150 \mathrm{~km} \mathrm{~s}^{-1}$ (Tremaine et al. 2002). Then, $n_{\mathrm{H}} \sim 2 \times 10^{8} \mathrm{~cm}^{-3}$ at $1 \mathrm{pc}$ and goes roughly as $r^{-2}$ at larger distances.

The important time scales in the accretion disk are the accretion time, in which the material at a radius $r$ is accreted into the black hole, and the dynamical time scale, in which the gas of density $\rho$ undergoes gravitational collapse. The accretion time is given by

$$
t_{\mathrm{acc}} \sim \frac{1}{\alpha_{\mathrm{vis}} \Omega}\left(\frac{r}{h}\right)^{2},
$$

where $\alpha_{\text {vis }}$ is the viscous parameter, which is usually $0.1-0.3$, and $h$ is the height at radius $r$. Here, we used the values $\alpha_{\text {vis }}=0.1$ and $h / r=0.1$. The dynamical time is similar to the crossing time in this case, which is the time for gas or stars to move from one side of the galaxy to the opposite side. In about every crossing time, in a turbulent medium, shock waves come through the medium. The dynamical time is given by

$$
t_{\mathrm{dyn}} \sim \frac{0.54}{\sqrt{G \rho}} .
$$

In the applications discussed below, we note that the accretion times $\left(1 \times 10^{7}-3 \times 10^{8} \mathrm{yr}\right)$ are three orders of magnitude greater than the dynamical times $\left(1 \times 10^{4}-3 \times 10^{5} \mathrm{yr}\right)$.

We modeled the chemistry of the midplane of the disk at distances of 3,10,30, and $50 \mathrm{pc}$ from the AGN core with two physical scenarios: one dominated by accretion time scales and the other by dynamical time scales. The temperatures and densities at these distances are listed in Tables 2 and 3 along with the relevant accretion and dynamical times, and the calculated fractional abundances with respect to $n_{\mathrm{H}}$ of some important species. We varied the cosmic-ray ionization rate from the standard galactic value of $\zeta_{\mathrm{H}_{2}}=1.3 \times 10^{-17} \mathrm{~s}^{-1}$ to $>5 \times 10^{-15} \mathrm{~s}^{-1}$ to see which value reproduces the observed abundances best. The value used for Tables 2 and 3 is $5 \times$ $10^{-15} \mathrm{~s}^{-1}$, which is best at $50 \mathrm{pc}$. We also used a visual extinction of $A_{V}=10$ and a radiation field with $G_{0}=1$, which results in almost no effect from the external UV photons since we assume that the column densities between OB stars are too high to allow a large-volume PDR. Different initial abundances were used in Tables 2 and 3. To obtain the results in Table 2, it was assumed that the molecules are being transported to the inner disk at the accretion time scale discussed above. For the $50 \mathrm{pc}$ result, we used the same initial abundances as in Table 1 except for hydrogen, which is in atomic form. Then we took the results at $50 \mathrm{pc}$ as the initial abundances for the $30 \mathrm{pc}$ calculation, and we ran the calculation for the time necessary for the material to
Table 2

Molecular Fractional Abundances with Respect to $n_{\mathrm{H}}$ at Accretion Times for Various Distances from the AGN Core at $\zeta=5 \times 10^{-15} \mathrm{~s}^{-1}$

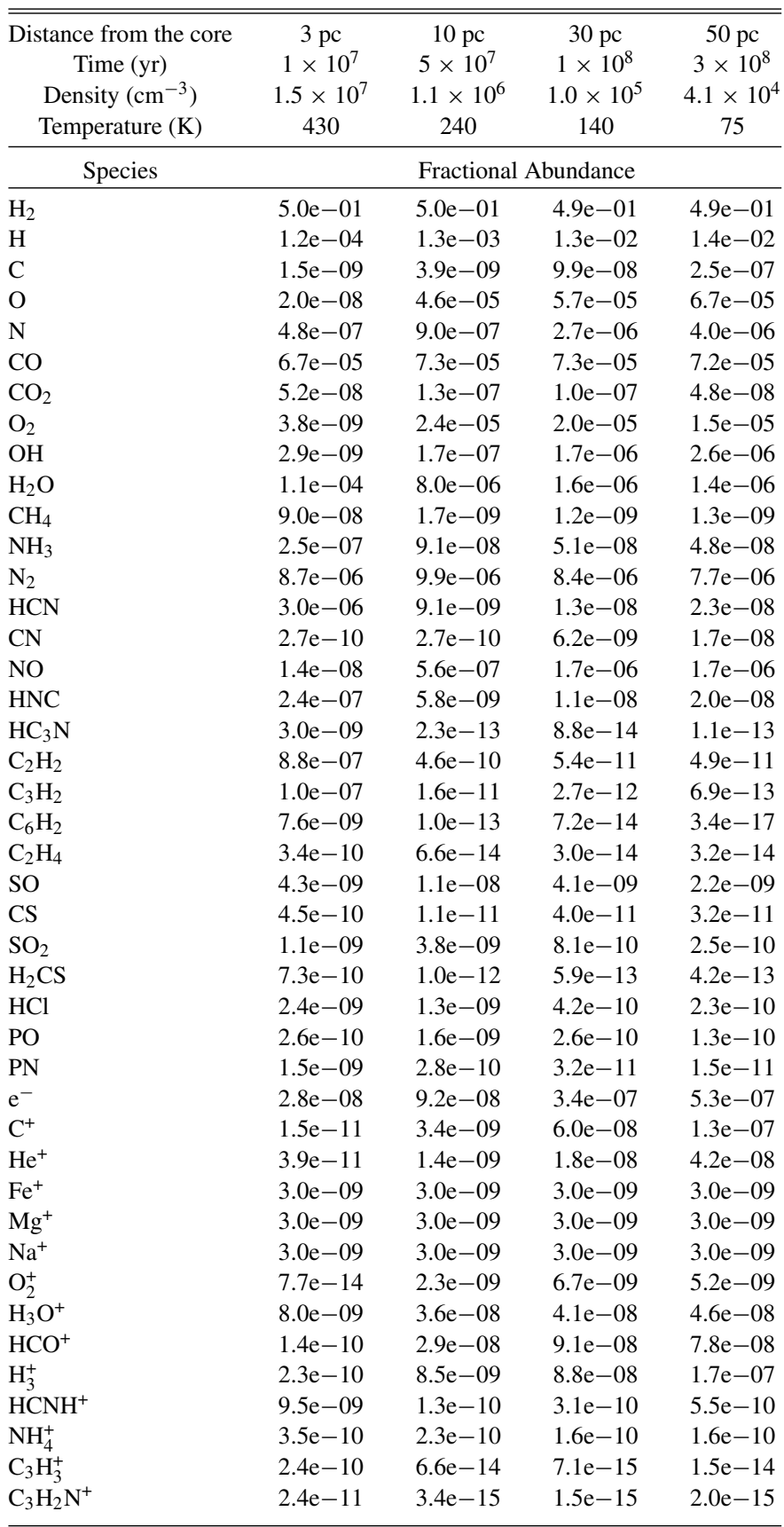

move inward to $30 \mathrm{pc}$, specifically $t_{\mathrm{acc}}(50 \mathrm{pc})-t_{\mathrm{acc}}(30 \mathrm{pc})$, which turns out to be longer than the time to reach steady state. Then we repeated the same process for the smaller radii. Note that for all radii less than $50 \mathrm{pc}$, the initial form of hydrogen is molecular. In Table 3, on the other hand, we made the assumption that shock waves exist on every dynamical time scale and leave little to no memory of abundances from the outer region. The likelihood that dissociative shocks occur at such a frequency, even with a very high ionization rate, is unclear, but we use this assumption just to determine how different the results of the dynamical model can be from those of the accretion case. We therefore started from atomic hydrogen or molecular hydrogen and the other initial abundances in Table 1, and ran the calculation for the dynamical timescale relevant to each radius $r$. Although the 
Table 3

Molecular Fractional Abundances with Respect to $n_{\mathrm{H}}$ at Dynamical Times for Various Distances from the AGN Core for $\zeta=5 \times 10^{-15} \mathrm{~s}^{-1}$ Starting with Molecular Hydrogen

\begin{tabular}{|c|c|c|c|c|}
\hline Distance from the core & $3 \mathrm{pc}$ & $10 \mathrm{pc}$ & $30 \mathrm{pc}$ & $50 \mathrm{pc}$ \\
\hline Time $(y r)$ & $1 \times 10^{4}$ & $5 \times 10^{4}$ & $1 \times 10^{5}$ & $3 \times 10^{5}$ \\
\hline Density $\left(\mathrm{cm}^{-3}\right)$ & $1.5 \times 10^{7}$ & $1.1 \times 10^{6}$ & $1.0 \times 10^{5}$ & $4.1 \times 10^{4}$ \\
\hline Temperature (K) & 430 & 240 & 140 & 75 \\
\hline Species & \multicolumn{4}{|c|}{ Fractional Abundance } \\
\hline$\overline{\mathrm{H}_{2}}$ & $5.0 \mathrm{e}-01$ & $5.0 \mathrm{e}-01$ & $4.9 \mathrm{e}-01$ & $4.9 \mathrm{e}-01$ \\
\hline $\mathrm{H}$ & $1.3 e-04$ & $1.3 e-03$ & $1.1 \mathrm{e}-02$ & $1.4 \mathrm{e}-02$ \\
\hline $\mathrm{C}$ & $1.3 e-10$ & $3.9 \mathrm{e}-09$ & $9.8 \mathrm{e}-08$ & $2.5 \mathrm{e}-07$ \\
\hline $\mathrm{O}$ & $8.9 \mathrm{e}-09$ & $4.6 e-05$ & $5.7 \mathrm{e}-05$ & $6.7 \mathrm{e}-05$ \\
\hline $\mathrm{N}$ & $1.1 \mathrm{e}-07$ & $9.0 \mathrm{e}-07$ & $2.7 e-06$ & $4.0 \mathrm{e}-06$ \\
\hline $\mathrm{CO}$ & $3.0 \mathrm{e}-05$ & $7.3 e-05$ & $7.3 e-05$ & $7.2 \mathrm{e}-05$ \\
\hline $\mathrm{CO}_{2}$ & $1.5 e-07$ & $1.3 e-07$ & $1.0 \mathrm{e}-07$ & $4.8 \mathrm{e}-08$ \\
\hline $\mathrm{O}_{2}$ & $4.7 e-07$ & $2.4 \mathrm{e}-05$ & $2.1 \mathrm{e}-05$ & $1.5 \mathrm{e}-05$ \\
\hline $\mathrm{OH}$ & $2.6 \mathrm{e}-09$ & $1.7 \mathrm{e}-07$ & $1.7 \mathrm{e}-06$ & $2.6 e-06$ \\
\hline $\mathrm{H}_{2} \mathrm{O}$ & $1.4 \mathrm{e}-04$ & $8.0 \mathrm{e}-06$ & $1.6 \mathrm{e}-06$ & $1.4 \mathrm{e}-06$ \\
\hline $\mathrm{CH}_{4}$ & $4.9 \mathrm{e}-07$ & $1.7 \mathrm{e}-09$ & $1.2 \mathrm{e}-09$ & $1.3 \mathrm{e}-09$ \\
\hline $\mathrm{NH}_{3}$ & $2.5 \mathrm{e}-07$ & $9.1 \mathrm{e}-08$ & $5.2 \mathrm{e}-08$ & $4.8 \mathrm{e}-08$ \\
\hline $\mathrm{N}_{2}$ & $7.4 \mathrm{e}-06$ & $9.9 \mathrm{e}-06$ & $8.4 \mathrm{e}-06$ & $7.7 \mathrm{e}-06$ \\
\hline $\mathrm{HCN}$ & $5.8 \mathrm{e}-06$ & $9.1 \mathrm{e}-09$ & $1.3 \mathrm{e}-08$ & $2.3 \mathrm{e}-08$ \\
\hline $\mathrm{CN}$ & $3.4 \mathrm{e}-10$ & $2.7 \mathrm{e}-10$ & $6.3 e-09$ & $1.7 \mathrm{e}-08$ \\
\hline NO & $1.4 \mathrm{e}-08$ & $5.6 e-07$ & $1.7 \mathrm{e}-06$ & $1.7 \mathrm{e}-06$ \\
\hline HNC & $2.9 \mathrm{e}-07$ & $5.8 \mathrm{e}-09$ & $1.1 \mathrm{e}-08$ & $2.0 \mathrm{e}-08$ \\
\hline $\mathrm{HC}_{3} \mathrm{~N}$ & $4.0 \mathrm{e}-08$ & $2.3 e-13$ & $9.0 \mathrm{e}-14$ & $1.1 \mathrm{e}-13$ \\
\hline $\mathrm{C}_{2} \mathrm{H}_{2}$ & $9.0 \mathrm{e}-06$ & $4.6 e-10$ & $5.5 e-11$ & $4.9 \mathrm{e}-11$ \\
\hline $\mathrm{C}_{3} \mathrm{H}_{2}$ & $6.3 e-07$ & $1.6 \mathrm{e}-11$ & $2.8 \mathrm{e}-12$ & $6.9 \mathrm{e}-13$ \\
\hline $\mathrm{C}_{6} \mathrm{H}_{2}$ & $1.3 e-06$ & $1.0 \mathrm{e}-13$ & $7.4 \mathrm{e}-14$ & $3.4 \mathrm{e}-17$ \\
\hline $\mathrm{C}_{2} \mathrm{H}_{4}$ & $3.5 \mathrm{e}-09$ & $6.6 e-14$ & $3.1 \mathrm{e}-14$ & $3.2 \mathrm{e}-14$ \\
\hline SO & $1.1 \mathrm{e}-08$ & $1.1 \mathrm{e}-08$ & $4.2 \mathrm{e}-09$ & $2.2 \mathrm{e}-09$ \\
\hline CS & $5.9 \mathrm{e}-10$ & $1.1 \mathrm{e}-11$ & $4.0 \mathrm{e}-11$ & $3.2 \mathrm{e}-11$ \\
\hline $\mathrm{SO}_{2}$ & $3.5 \mathrm{e}-09$ & $3.8 \mathrm{e}-09$ & $8.3 e-10$ & $2.5 \mathrm{e}-10$ \\
\hline $\mathrm{H}_{2} \mathrm{CS}$ & $1.5 \mathrm{e}-09$ & $1.0 \mathrm{e}-12$ & $5.9 \mathrm{e}-13$ & $4.2 \mathrm{e}-13$ \\
\hline $\mathrm{HCl}$ & $2.4 \mathrm{e}-09$ & $1.3 e-09$ & $4.2 \mathrm{e}-10$ & $2.3 e-10$ \\
\hline PO & $6.8 \mathrm{e}-10$ & $1.6 e-09$ & $2.6 \mathrm{e}-10$ & $1.3 e-10$ \\
\hline PN & $7.5 e-10$ & $2.8 \mathrm{e}-10$ & $3.3 e-11$ & $1.5 \mathrm{e}-11$ \\
\hline $\mathrm{e}^{-}$ & $2.8 \mathrm{e}-08$ & $9.2 \mathrm{e}-08$ & $3.4 \mathrm{e}-07$ & $5.3 e-07$ \\
\hline $\mathrm{C}^{+}$ & $4.4 \mathrm{e}-12$ & $3.4 \mathrm{e}-09$ & $6.0 \mathrm{e}-08$ & $1.3 \mathrm{e}-07$ \\
\hline $\mathrm{He}^{+}$ & $3.2 \mathrm{e}-11$ & $1.4 \mathrm{e}-09$ & $1.8 \mathrm{e}-08$ & $4.2 \mathrm{e}-08$ \\
\hline $\mathrm{Fe}^{+}$ & $3.0 \mathrm{e}-09$ & $3.0 \mathrm{e}-09$ & $3.0 \mathrm{e}-09$ & $3.0 \mathrm{e}-09$ \\
\hline $\mathrm{Mg}^{+}$ & $3.0 \mathrm{e}-09$ & $3.0 \mathrm{e}-09$ & $3.0 \mathrm{e}-09$ & $3.0 \mathrm{e}-09$ \\
\hline $\mathrm{Na}^{+}$ & $3.0 \mathrm{e}-09$ & $3.0 \mathrm{e}-09$ & $3.0 \mathrm{e}-09$ & $3.0 \mathrm{e}-09$ \\
\hline $\mathrm{O}_{2}^{+}$ & $6.6 e-12$ & $2.3 e-09$ & $6.5 e-09$ & $5.2 \mathrm{e}-09$ \\
\hline $\mathrm{H}_{3} \mathrm{O}^{+}$ & $5.1 \mathrm{e}-09$ & $3.6 \mathrm{e}-08$ & $4.1 \mathrm{e}-08$ & $4.6 e-08$ \\
\hline $\mathrm{HCO}^{+}$ & $3.7 \mathrm{e}-11$ & $2.9 \mathrm{e}-08$ & $9.1 \mathrm{e}-08$ & $7.8 \mathrm{e}-08$ \\
\hline $\mathrm{H}_{3}{ }^{+}$ & $1.9 \mathrm{e}-10$ & $8.5 e-09$ & $8.8 \mathrm{e}-08$ & $1.7 \mathrm{e}-07$ \\
\hline $\mathrm{HCNH}^{+}$ & $1.1 \mathrm{e}-08$ & $1.3 \mathrm{e}-10$ & $3.1 \mathrm{e}-10$ & $5.5 e-10$ \\
\hline $\mathrm{NH}_{4}^{+}$ & $3.0 \mathrm{e}-10$ & $2.3 e-10$ & $1.6 \mathrm{e}-10$ & $1.6 \mathrm{e}-10$ \\
\hline $\mathrm{C}_{3} \mathrm{H}_{3}^{+}$ & $9.3 e-10$ & $6.6 e-14$ & $7.8 \mathrm{e}-15$ & $1.5 \mathrm{e}-14$ \\
\hline $\mathrm{C}_{3} \mathrm{H}_{2} \mathrm{~N}^{+}$ & $2.8 \mathrm{e}-10$ & $3.4 \mathrm{e}-15$ & $1.5 \mathrm{e}-15$ & $2.0 \mathrm{e}-15$ \\
\hline
\end{tabular}

use of molecular hydrogen might seem artificial, its widespread abundance in the interstellar medium in regions where other molecular development is slight makes the case for using it an arguable one.

It is likely that the elemental abundances are different from our values, which are mainly derived for dense clouds in our Galaxy. There might be less depletion of metals on grains, for example, and super-solar metallicity has been observed in some AGNs (Mathur \& Fields 2009); both of these factors increase the metal abundance. We ran the code with higher metal abundances and obtained similar results closer to the center of the AGN, although differences are greater for colder regions farther out. The effect of shock waves was also ignored, other than to refresh the material at the dynamical time scales. There are other physical processes, such as the emission of UV photons from star formation and the generation of cosmic rays from supernovae, which change the ionization conditions, at least locally. It is also important to note that our results here are only for the midplane of the disk. At larger heights, where the medium is more tenuous, X-rays from the AGN core can penetrate through the disk, and there will be ionizing effects on the chemistry. The effects of UV photons and cosmic rays must also be different at different heights. Actual observations in most cases are through integrated columns involving different heights. We would thus expect that, as in a protoplanetary disk, a model solely of the midplane might miss the radicals associated with higher elevations. We will postpone a consideration of all these parameters to a more detailed discussion (N. Harada et al. 2010, in preparation).

\subsection{Results and Implications from Current Observations}

Since a high value of the ionization rate, $\zeta_{\mathrm{H}_{2}}=5 \times 10^{-15} \mathrm{~s}^{-1}$, was used in order to achieve best agreement with observation at $50 \mathrm{pc}$, most species achieve steady-state abundances relatively quickly, and there are no significant differences for these species between the accretion case starting with atomic hydrogen and the dynamical case starting with molecular hydrogen. The general trend for larger species is to show a significant increase in abundance at the inner radii where higher temperatures prevail. Some complex molecules, such as $\mathrm{C}_{2} \mathrm{H}_{2}$ or $\mathrm{HC}_{3} \mathrm{~N}$, have a peak before steady state is reached, and are enhanced by $\approx$ an order of magnitude in the dynamical case as compared with the accretion case at $3 \mathrm{pc}$. When the initial form of hydrogen in the dynamic case is atomic, the abundances of molecules at $50 \mathrm{pc}$ are orders of magnitude lower except for $\mathrm{CO}$ and $\mathrm{CN}$, which have similar abundances to the other cases. At smaller radii, the initial form of hydrogen in the dynamic model is unimportant because, at the higher densities, the conversion of $\mathrm{H}$ into $\mathrm{H}_{2}$ occurs quickly.

How do our preliminary results compare with observations? As a cautionary note, it must be remembered that observed intensities can reflect both molecular abundances and radiative transfer considerations. Our comparison will be limited to the AGN-dominant galaxy NGC 1068, where actual column densities have been obtained.

In particular, Usero et al. (2004) obtained the following column density ratios for $\mathrm{HCN}, \mathrm{HCO}^{+}, \mathrm{CN}$, and $\mathrm{CS}$ with respect to $\mathrm{CO}: \mathrm{HCN} / \mathrm{CO}=1 \times 10^{-3}, \mathrm{CN} / \mathrm{CO}=(1-6) \times 10^{-3}, \mathrm{HCO}^{+} /$ $\mathrm{CO}=(0.8-2) \times 10^{-3}$, and $\mathrm{CS} / \mathrm{CO}=2.5 \times 10^{-4}$. They estimated the source size to have a radius of about 1.1 arcsec, which corresponds to about $80 \mathrm{pc}$. Our largest distance is $50 \mathrm{pc}$, so we use this distance for comparison. Note that gas-phase models at larger distances are inaccurate because the temperature is low enough for significant ice accumulation. Also, unless we mention a difference, there is none between our computed accretion and dynamical models, when the latter uses molecular hydrogen as the initial condition.

At $50 \mathrm{pc}$, our value for $\mathrm{CN} / \mathrm{CO}$ is a factor of $4-25$ below the observed value, although we are much closer to the lower bound suggested in a more recent work by Garcia-Burillo et al. (2010). HCN/CO is underproduced by a factor of 3 compared with the value by Usero et al. (2004), while our calculated value for $\mathrm{HCO}^{+} / \mathrm{CO}, 1.1 \times 10^{-3}$, is within their observed range of abundance ratios. The worst disagreement occurs for $\mathrm{CS} / \mathrm{CO}$, where our calculated value of $4 \times 10^{-7}$ is $500 \times$ lower than the observed one, possibly reflecting our use of too low an abundance of sulfur. For the dynamical case, the abundances 
of the observed species can be reproduced to a similar degree with somewhat lower ionization rates, if the time scale is also shortened. Also, if the temperature is raised by a factor of 2 from the values used here in both the accretion and dynamical models, little difference is seen at $50 \mathrm{pc}$.

Other column density ratios remain an important observational tool for NGC 1068. Pérez-Beaupuits et al. (2009) observed high $J$ lines for $\mathrm{HCN}, \mathrm{HNC}, \mathrm{HCO}^{+}$, and $\mathrm{CN}$ and determined column density ratios of $\mathrm{N}(\mathrm{CN}) / \mathrm{N}(\mathrm{HCN}) \sim 0.7-0.9$ and $\mathrm{N}(\mathrm{HNC}) / \mathrm{N}(\mathrm{HCN}) \sim 0.10-0.18$. The estimate for $\mathrm{N}(\mathrm{HNC}) /$ $\mathrm{N}(\mathrm{HCN})$ is in agreement with that based on earlier lower $J$ observations (Pérez-Beaupuits et al. 2007). The emission is from a source of size $\sim 1$ ", which corresponds to $72 \mathrm{pc}$, which, once again corresponds best with our $50 \mathrm{pc}$ case. At this distance, our results show the $\mathrm{CN} / \mathrm{HCN}$ abundance ratio to be 0.7 , a value in good agreement with observation. For the dynamical case starting from atomic hydrogen, the $\mathrm{CN} / \mathrm{HCN}$ abundance ratio is much too high. Our $\mathrm{HNC} / \mathrm{HCN}$ ratios $(\approx 1)$ seem to be a factor of 4-8 high, although they do decrease at distances closer to the AGN core. We also note that the $50 \mathrm{pc}$ region is not the region of high temperature that this paper is primarily concerned with. We expect that those portions of the disk closer to the AGN will have very different physical conditions, and it will be very exciting to have finer resolution observations by ALMA.

\section{SUMMARY}

We have developed an extended gas-phase chemical network including complex molecules that can be used for a source at temperatures up to $800 \mathrm{~K}$ with the assumption that the gas and dust temperatures are the same. To avoid the need for the inclusion of grain-surface ice chemistry, dust temperatures in excess of $100 \mathrm{~K}$ are implied so that the range of the model is $100 \mathrm{~K}<T<800 \mathrm{~K}$. The network has been used to study, in a preliminary manner, the chemistry in molecular disks of AGNs under various conditions. Given the relaxed constraints on which classes of reactions are important at high temperatures, we note that it may be necessary to include additional reactions if the physical conditions warrant it. Moreover, there are many significant reactions in our extended system for which there is little information available, including on occasion even thermodynamic data. Sensitivity analyses may help to direct laboratory and theoretical investigations toward the most important yet poorly understood systems. With the advent of ALMA and other next generation telescopes, there will be many galactic and extragalactic high-temperature sources that we will be able to see with much higher spatial resolution. Our model will be useful for the analysis of these sources.

E.H. acknowledges the support of the National Science Foundation for his astrochemistry program through grant AST0702876 and his program in chemical kinetics through the Center for the Chemistry of the Universe. He also acknowledges support from NASA NAI for studies in the evolution of preplanetary matter. V.W. thanks the French program PCMI for partial support of this work. We thank the following individuals for their help with various aspects of this research: M. Agúndez, J. Cernicharo, J. Goicoechea, G. Pineau des Forêts, P. Rimmer, E. Roueff, T. Thompson, and D. Woon.

\section{REFERENCES}

Aalto, S. 2008, Ap\&SS, 313, 273

Agúndez, M., Cernicharo, J., \& Goicoechea, J. R. 2008, A\&A, 483, 831
Anicich, V. G. 1993, J. Phys. Chem. Ref. Data, 22, 1469

Bates, D. R., \& Herbst, E. 1988, in Rate Coefficients in Astrochemistry, ed. T. J. Millar \& D. A. Williams (Dordrecht: Kluwer), 17

Bergin, E. A., Neufeld, D. A., \& Melnick, G. J. 1998, ApJ, 499, 777

Burke, J. R., \& Hollenbach, D. J. 1983, ApJ, 265, 223

Cazaux, S., Caselli, P., Tielens, A. G. G. M., LeBourlot, J., \& Walmsley, C. M. 2005, J. Phys. Conf. Ser., 6, 155

Cuppen, H. M., \& Hornekær, L. 2008, J. Chem. Phys., 128, 174707

Draine, B. T. 1978 , ApJS, 36, 595

Elitzur, M., \& de Jong, T. 1978, A\&A, 67, 323

Garcia-Burillo, S., et al. 2010, arXiv: 1005.1263

Garrod, R. T., Weaver, S. L. W., \& Herbst, E. 2008, ApJ, 682, 283

Gerlich, D., \& Kaefer, G. 1989, ApJ, 347, 849

Glassgold, A. E., Meijerink, R., \& Najita, J. R. 2009, ApJ, 701, 142

Gorti, U., \& Hollenbach, D. 2008, ApJ, 683, 287

Graedel, T. E., Langer, W. D., \& Frerking, M. A. 1982, ApJS, 48, 321

Greenhill, L. J., \& Gwinn, C. R. 1997, Ap\&SS, 248, 261

Herbst, E. 1985, ApJ, 291, 226

Herbst, E., \& Klemperer, W. 1973, ApJ, 185, 505

Herbst, E., \& Leung, C. M. 1986, ApJ, 310, 378

Herbst, E., \& Millar, T. 2008, in Low Temperatures and Cold Molecules, ed. I. W. M. Smith (London: Imperial College Press), 1

Hollenbach, D., \& McKee, C. F. 1979, ApJS, 41, 555

Jaffe, W., et al. 2004, Nature, 429, 47

Koda, J., Okuda, T., Nakanishi, K., Kohno, K., Ishizuki, S., Kuno, N., \& Okumura, S. K. 2005, A\&A, 431, 887

Le Petit, F., Nehmé, C., Le Bourlot, J., \& Roueff, E. 2006, ApJS, 164, 506

Lee, H.-H., Herbst, E., Pineau des Forêts, G., Roueff, E., \& Le Bourlot, J. 1996, A\&A, 311, 690

Lucero, D. M., \& Young, L. M. 2007, AJ, 134, 2148

Maergoiz, A. I., Nikitin, E. E., \& Troe, J. 2009, Int. J. Mass Spectrom., 280, 42

Mallard, W. G., et al. 2010, NIST Chemistry WebBook, NIST Standard Reference Database 69, http://webbook.nist.gov/chemistry/

Maloney, P. R., Hollenbach, D. J., \& Tielens, A. G. G. M. 1996, ApJ, 466, 561

Markwick, A. J., Ilgner, M., Millar, T. J., \& Henning, T. 2002, A\&A, 385, 632

Martin, C. L., \& Kennicutt, R. C., Jr. 2001, ApJ, 555, 301

Mason, R. E., Geballe, T. R., Packham, C., Levenson, N. A., Elitzur, M., Fisher, R. S., \& Perlman, E. 2006, ApJ, 640, 612

Mathur, S., \& Fields, D. 2009, in AIP Conf. Proc., Vol. 1135, Future Directions in Ultraviolet Spectroscopy, ed. M. E. van Steenberg, et al. (Melville, NY: AIP), 209

McEwan, M. J., Scott, G. B. I., Adams, N. G., Babcock, L. M., Terzieva, R., \& Herbst, E. 1999, ApJ, 513, 287

Meijerink, R. 2006, $\mathrm{PhD}$ thesis, Leiden Univ.

Meijerink, R., \& Spaans, M. 2005, A\&A, 436, 397

Meijerink, R., Spaans, M., \& Israel, F. P. 2007, A\&A, 461, 793

Nenkova, M., Sirocky, M. M., Ivezić, Ž., \& Elitzur, M. 2008, ApJ, 685, 147

Neufeld, D. A., \& Dalgarno, A. 1989, ApJ, 340, 869

Nomura, H., Aikawa, Y., Nakagawa, Y., \& Millar, T. J. 2009, A\&A, 495, 183

Pérez-Beaupuits, J. P., Aalto, S., \& Gerebro, H. 2007, A\&A, 476, 177

Pérez-Beaupuits, J. P., Spaans, M., van der Tak, F. F. S., Aalto, S., García-Burillo, S., Fuente, A., \& Usero, A. 2009, A\&A, 503, 459

Raban, D., Jaffe, W., Röttgering, H., Meisenheimer, K., \& Tristram, K. R. W. 2009, MNRAS, 394, 1325

Rebrion, C., Marquette, J. B., Rowe, B. R., \& Clary, D. C. 1988, Chem. Phys. Lett., 143, 130

Röllig, M., et al. 2007, A\&A, 467, 187

Schinnerer, E., Eckart, A., Tacconi, L. J., Genzel, R., \& Downes, D. 2000, ApJ, 533,850

Silk, J. 1997, ApJ, 481, 703

Stäuber, P., Doty, S. D., van Dishoeck, E. F., \& Benz, A. O. 2005, A\&A, 440, 949

Su, T., \& Chesnavich, W. J. 1982, J. Chem. Phys., 76, 5183

Tacconi, L. J., Genzel, R., Blietz, M., Cameron, M., Harris, A. I., \& Madden, S. 1994, ApJ, 426, L77

Thompson, T. A., Quataert, E., \& Murray, N. 2005, ApJ, 630, 167

Toomre, A. 1964, ApJ, 139, 1217

Tremaine, S., et al. 2002, ApJ, 574, 740

Usero, A., García-Burillo, S., Fuente, A., Martín-Pintado, J., \& RodríguezFernández, N. J. 2004, A\&A, 419, 897

Vollmer, B., Beckert, T., \& Davies, R. I. 2008, A\&A, 491, 441

Wakelam, V., et al. 2010, Space Sci. Rev., submitted

Woitke, P., Kamp, I., \& Thi, W.-F. 2009, A\&A, 501, 383

Woods, P. M., \& Willacy, K. 2009, ApJ, 693, 1360

Woon, D. E., \& Herbst, E. 2009, ApJS, 185, 273 